\title{
Gas physical conditions and kinematics of the giant outflow Ou4 ${ }^{\star}$
}

\author{
Romano L. M. Corradi ${ }^{1,2}$, Nicolas Grosso ${ }^{3, \star \star}$, Agnès Acker $^{3}$, Robert Greimel ${ }^{4}$, and Patrick Guillout ${ }^{3}$ \\ ${ }^{1}$ Instituto de Astrofísica de Canarias, 38200 La Laguna, Tenerife, Spain \\ e-mail: rcorradi@iac.es \\ 2 Departamento de Astrofísica, Universidad de La Laguna, 38206 La Laguna, Tenerife, Spain \\ 3 Observatoire Astronomique de Strasbourg, Université de Strasbourg, CNRS, UMR 7550, 11 rue de l'Université, 67000 Strasbourg, \\ France \\ ${ }^{4}$ IGAM, Institut für Physik, Universität Graz, Universitätsplatz 5/II, 8010 Graz, Austria
}

Received 20 September 2013 / Accepted 8 July 2014

\begin{abstract}
Context. The recently discovered bipolar outflow Ou4 has a projected size of more than one degree in the plane of the sky. It is apparently centred on the young stellar cluster - whose most massive representative is the triple system HR 8119 - inside the H II region Sh 2-129. The driving source, the nature, and the distance of Ou4 are not known.

Aims. The basic properties of Ou4 and its environment are investigated to shed light on the origin of this remarkable outflow.

Methods. Deep narrow-band imagery of the whole nebula at arcsecond resolution was obtained to study the details of its morphology. Long-slit spectroscopy of the bipolar lobe tips was secured to determine the gas ionisation mechanism, physical conditions, and line-of-sight velocities. An estimate of the proper motions at the tip of the south lobe using archival plate images was attempted. The existing multi-wavelength data for Sh 2-129 and HR 8119 were also comprehensively reviewed.

Results. The observed morphology of Ou4, its emission-line spatial distribution, line flux ratios, and the kinematic modelling developed adopting a bow-shock parabolic geometry, illustrate the expansion of a shock-excited fast collimated outflow. The observed radial velocities of Ou4 and its reddening are consistent with those of Sh 2-129 and HR 8119. The improved determination of the distance to HR 8119 (composed of two B0 V and one B0.5 V stars) and Sh 2-129 is 712 pc. We identify in WISE images at $22 \mu \mathrm{m}$ an emission bubble of $5^{\prime}$ radius ( $1 \mathrm{pc}$ at the distance above) emitted by hot (107 K) dust grains, located inside the central part of Ou4 and corresponding to several [O III] emission features of Ou4.

Conclusions. The apparent position of Ou4 and the properties studied in this work are consistent with the hypothesis that Ou4 is located inside the Sh 2-129 H II region, suggesting that it was launched some 90000 yr ago by HR 8119. The outflow total kinetic energy is estimated to be $\approx 4 \times 10^{47}$ ergs. However, we cannot rule out the alternative possibility that Ou 4 is a bipolar planetary nebula or the result of an eruptive event on a massive AGB or post-AGB star not yet identified.
\end{abstract}

Key words. ISM: individual objects: Ou4 - ISM: jets and outflows - H II regions - planetary nebulae: general - stars: early-type stars: winds, outflows

\section{Introduction}

The remarkable nebula Ou4 was discovered by the French amateur astronomer Nicolas Outters while imaging the Sh 2-129 nebula in June 2011 by means of a $12.5 \mathrm{~h}$ CCD exposure with an F/5 $106 \mathrm{~mm}$-diameter refractor (resulting in a plate scale of $\left.3^{\prime \prime} .5 \mathrm{pix}^{-1}\right)$ and a narrow ( $\left.30 \AA F W H M\right)$ [O III] filter. It is a clear example of the relevant science that can be done with very small telescopes: the long integrations that can be obtained by dedicated amateur astronomers allow very faint detection levels to be reached, which are sometimes difficult to achieve with professional telescopes given the limited amount of time available ${ }^{1}$.

The nebula Ou4 was introduced and discussed by Acker et al. (2012). Its highly bipolar morphology is mainly visible in the [O III] $5007 \AA$ light. The total length of the nebula is 1.2 on the

\footnotetext{
* Based on observations, obtained under Director's Discretionary Time of the Spanish Instituto de Astrofísica de Canarias, with the $2.5 \mathrm{~m}$ INT and the $4.2 \mathrm{~m}$ WHT operated on the island of La Palma by the Isaac Newton Group of Telescopes in the Spanish Observatorio of the Roque de Los Muchachos.

1 Note that the detection limit per resolution element of an extended, uniform source does not depend on the telescope size, but only on its focal ratio.
}

sky. The driving source, the nature, and the distance of Ou4 are not known. Acker et al. (2012) discussed several possibilities, including that $\mathrm{Ou} 4$ might be a very nearby planetary nebula (PN).

Motivated by the impressive size and remarkable morphology of Ou4, we have obtained new optical images and spectra with the aim to shed light on its origin. In Sect. 2, we describe the relative position in the sky of $\mathrm{Ou} 4$ and the $\mathrm{HII}$ region Sh 2-129, and discuss the distance to the latter. Our imaging and spectroscopic observations are presented in Sect. 3. We analyse these results in Sect. 4, and discuss the nature of Ou4 in Sect. 5.

\section{Ou4 and the Sh 2-129 nebula}

\subsection{Multi-wavelength view of the Sh 2-129 nebula}

A multi-wavelength view of the Sh 2-129 H II region is presented in Fig. 1. The $R$-band DDS2 image mainly shows the $\mathrm{H} \alpha$ line emission. This defines the boundary of this ionised region, which consists of a bright semicircle on the east side and a fainter elongated bubble protruding towards the north-west direction. This morphology is typical of an evolved H II region in the Champagne phase (Tenorio-Tagle 1979): the ionising flux of a hot young star originally embedded near the edge of a 


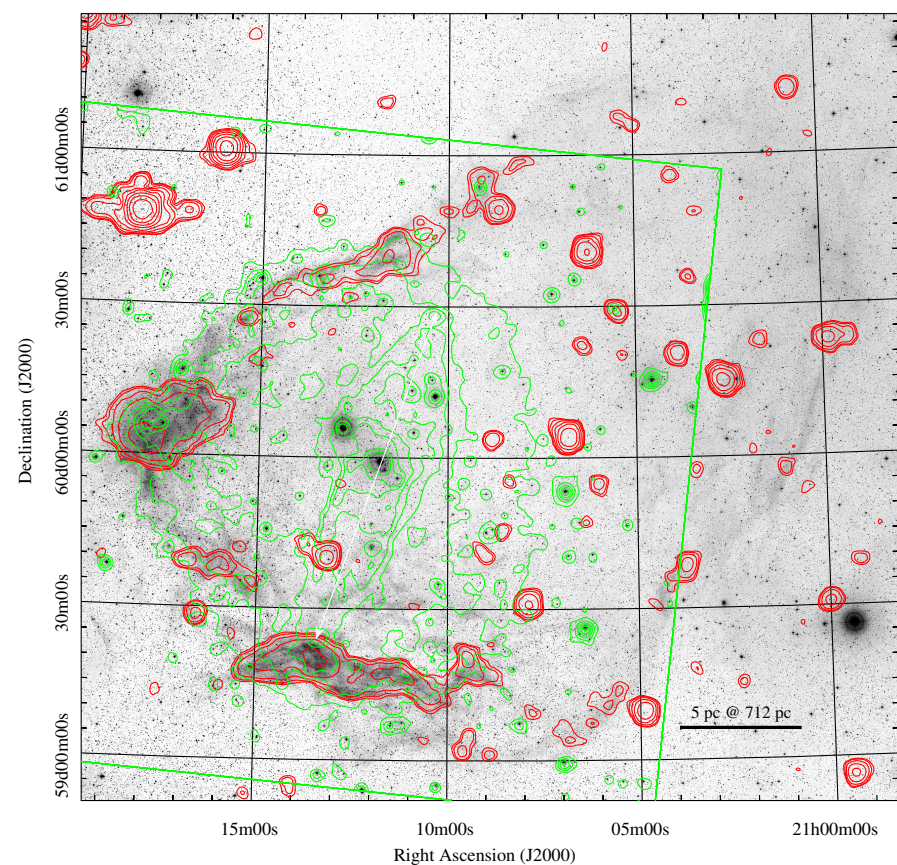

Fig. 1. Blister H II region Sh2-129 and Ou4. The DSS2 R-band image is shown in greyscale in the background. The red contour map is the Green Bank $6 \mathrm{~cm}$ survey (Condon et al. 1994) using 10 contours from 0.01 to $2.8 \mathrm{Jy} /$ beam with a linear step. The green contour map is a smoothed and thresholded [O III] image. The image and contour-map scales are logarithmic. The white arrowed line has a length of 1.2 and indicates the position of the giant outflow Ou4. HR 8119, the young massive star that ionises Sh 2-129, is close to the middle of this line.

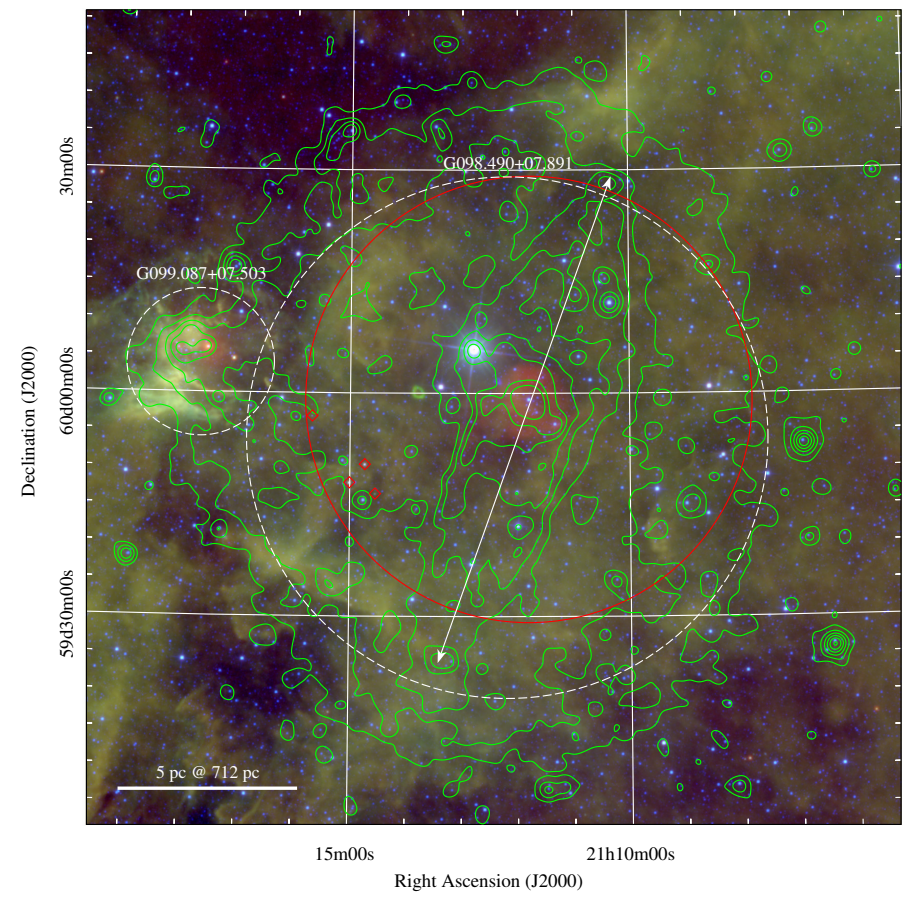

Fig. 2. Mid-infrared image of Sh 2-129 obtained from the WISE AllSky Atlas. Blue, green, and red represent the WISE infrared bands $W 2$, $W 3$, and $W 4$ centred at $4.6,12$, and $22 \mu \mathrm{m}$ filters, respectively. The green contour map is the same [O III] image shown in Fig. 1. The dashed white circles indicate the two (radio-quiet) H II regions in the WISE catalogue of Galactic H II regions (Anderson et al. 2014). The red diamonds mark the classical T Tauri star candidates that we have identified inside the $30^{\prime}$-radius red circle centred on HR 8119 (see labels in Fig. 3).

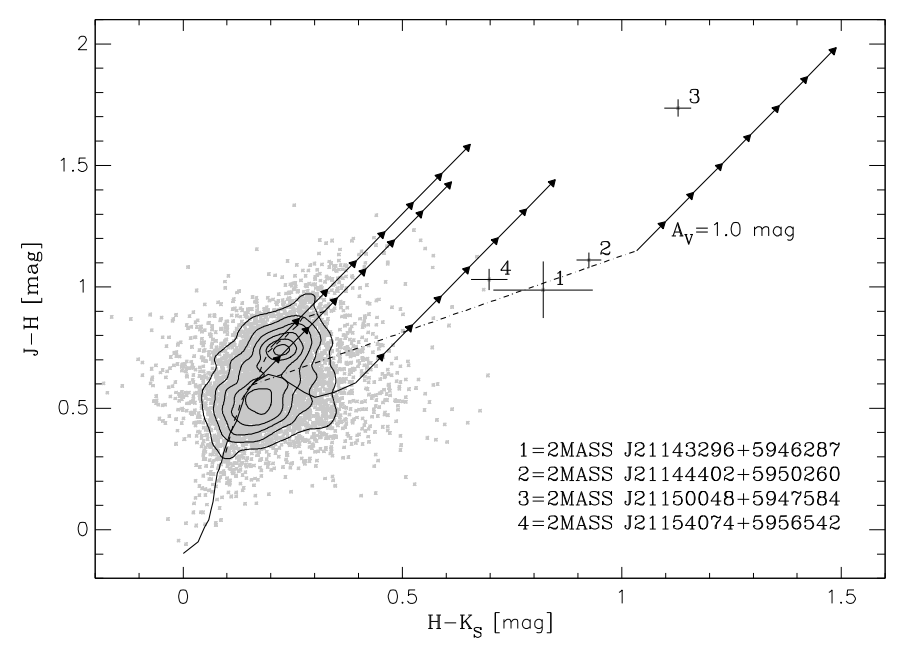

Fig. 3. Near-infrared colour-colour diagram of sources around HR 8119. Grey asterisks are 2MASS sources with good photometry located at a maximum angular distance of $30^{\prime}$ from HR 8119 (see red circle in Fig. 2). Contours indicate the source density. The continuous and dashed lines are the loci of main-sequence and giant stars of Bessell \& Brett (1988). The dotted-dashed line is the locus of the classical T Tauri stars (Meyer et al. 1997). The arrows show the extinction vectors (Cohen et al. 1981). The classical T Tauri star candidates are labelled.

molecular cloud produced an expanding spherical H II region that pierced the cloud surface producing a flow of ionised gas (e.g., Yorke et al. 1983).

The Green Bank $6 \mathrm{~cm}$ survey (Condon et al. 1994; Fig. 1) shows the free-free continuum emission from the border of this blister H II region. In a large field-of-view [O III] image of Sh $2-129^{2}$ the [O III] emission of Ou4 appears as superposed and centred on the fainter emission from the hot gas that fills the semi-spherical H II region of Sh 2-129 and cools within the Champagne flow.

Infrared images obtained with the Wide-field Infrared Survey Explorer (WISE; Wright et al. 2010) unveil the dust emission in this area (Fig. 2). Bright dust pillars are present at the eastern border of the bubble, but they are associated with a smaller H II region according to Anderson et al. (2014). This small H II region and dust pillars are typical of star-forming regions. This star-forming region may have been triggered by the expansion of Sh 2-129. In the central part of Sh 2-129 an emission bubble of $5^{\prime}$ radius is detected at $22 \mu \mathrm{m}$. Such an emission is usually observed inside H II regions where the central hot star heats dust grains (e.g., Deharveng et al. 2010); for instance, the dust pillars of the smaller H II region are also surrounded by $22 \mu \mathrm{m}$ emission. The morphology of the Sh 2-129 mid-infrared bubble and its relation with Ou4 is discussed in Sect. 4.5.

We used 2MASS (Skrutskie et al. 2006) to identify young star candidates in the central part of Sh 2-129, in a radius of $30^{\prime}$ from HR 8119 . We selected only sources with good photometry (AAA flag) to build a colour-colour near-infrared diagram in the 2MASS photometric system (Fig. 3). The comparison with

2 The original image was obtained by another French amateur astronomer, Stéphane Zoll, on August 2012 by means of a 7.3 h CCDexposure with a F/3.6 $106 \mathrm{~mm}$-diameter refractor (plate scale of $\left.4^{\prime \prime} \mathrm{pix}^{-1}\right)$ and a narrow ( 50 $\mathrm{\AA} F H M$ ) [O III] filter, and posted on www. astrosurf.com. We used Aladin (Bonnarel et al. 2000) to register it to the DSS2 image. 
the locus of classical $\mathrm{T}$ Tauri stars (CTTSs) in this diagram ${ }^{3}$ allowed us to identify four CTTS candidates by their infrared excess. There is no source with stronger infrared excess in this area, meaning, there are no protostars. These CTTS candidates are not located in the vicinity of HR 8119 , but close to the east border of our selection area; one object has an optical extinction of about 7 mag, whereas the other sources do not have an optical extinction larger than 1 mag.

\subsection{Distance to HR 8119 and Sh 2-129}

Georgelin \& Georgelin (1970) identified the hot (spectral type B0 V, Hiltner 1956) star HR 8119 (also known as HD 202214) as the ionising source of Sh 2-129. Consequently, HR 8119 and Sh 2-129 must be located at the same distance. We also note that HR 8119 is the star with the earliest spectral type in the stellar group that is visible at the centre of this blister $\mathrm{H}$ II region according to SIMBAD. This young star is a member of the Cepheus OB2 massive stars association (see, e.g., Fig. 3 of Patel et al. 1998) $)^{4}$. HR 8119 also lies in the central part of Ou4, as noted by Acker et al. (2012).

Acker et al. (2012) estimated the distance of HR 8119 to be $\sim 870 \mathrm{pc}$ by assuming no foreground extinction and an absolute magnitude for main-sequence stars, but the distance most often used in the literature for Sh 2-129 is 400 pc. This latter value is the spectrophotometric distance derived by Georgelin \& Georgelin (1970) assuming that HR 8119 is a single star. However, HR 8119 is a triple system, where the separations obtained from speckle interferometry between the primary and the $\mathrm{Ab}$ and B components were 0.'045 and 1.'021 at epoch 2005.8654 (Mason et al. 2009). We improved the distance determination by combining the dynamical mass of HR 8119A with evolutionary tracks of massive stars with solar metallicity (Bertelli et al. 1994) ${ }^{5}$. The orbital elements of the HR 8119Aa and $\mathrm{Ab}$ components were published by Zirm \& Rica (2012) and flagged as reliable in the Sixth Catalog of Orbits of Visual Binary Stars ${ }^{6}$. From the orbit semi-major axis $\left(a=0{ }^{\prime} 066\right)$ and the period $(P=56.93 \mathrm{yr})$, the following dynamical mass is obtained for HR 8119A: $M_{\mathrm{dyn}} / M_{\odot}=32.1 \times(P / 56.93 \mathrm{yr})^{-2} \times$ $\left(a / 0{ }^{\prime} 066\right)^{3} \times(d / 712 \mathrm{pc})^{3}$, where $d$ is the distance to HR 8119 . The following observational constraints are used: the spectral type B0 V for the primary component, which corresponds to an effective temperature of $31500 \mathrm{~K}$ (Pecaut \& Mamajek 2013); the differential $V$-band magnitude between the $\mathrm{Ab}$ and Aa components $(0.6 \mathrm{mag})$ in The Washington Visual Double Star Catalog (Mason et al. 2001); and the Tycho-2 magnitudes of HR 8119A and $\mathrm{B}, B_{\mathrm{T}}=6.151$ and $6.864 \mathrm{mag}$, and $V_{\mathrm{T}}=6.092$ and $6.768 \mathrm{mag}$, respectively (Høg et al. 2000), which are converted into $B=6.132$ and $6.838 \mathrm{mag}$, and $V=6.085$ and $6.757 \mathrm{mag}$, respectively, using the filter transformations of Mamajek et al. (2002). Comparison of these photometric data with the evolutionary tracks provides a best-fit age of 3.7 Myr, which gives a

\footnotetext{
3 We use the colour transformations for the final 2MASS data release at http://www.astro.caltech.edu/ jmc/2mass/v3/ transformations

4 Note in particular that HR 8119 cannot belong to the Trumpler 37 stellar cluster, which is located inside the IC $1396 \mathrm{H}$ II region (also known as Sh 2-131) at an angular distance of 4.3 east of Sh 2-129.

5 The CMD 2.5 web interface available at http://stev.oapd. inaf.it/cgi-bin/cmd was used.

6 http://WwW . usno.navy.mil/USNO/astrometry/

optical-IR-prod/wds/orb6
}

total mass of $32.0 M_{\odot}$ for HR $8119 \mathrm{~A}^{7}$, a distance of $712 \mathrm{pc}$, and a foreground visual extinction of $1.1 \mathrm{mag}$. The latter is consistent with the computed colour excess of HR 8119B (adopting $R_{V}=$ 3.1). The age determination is consistent with the rather evolved evolutionary status of this HII region and the lack of 2MASS sources (Skrutskie et al. 2006) with near-infrared excess (i.e., accretion circumstellar disc) in the vicinity of HR 8119. Therefore, we adopt a distance of 712 pc to HR 8119 and Sh 2-129.

\section{Observations}

\subsection{Imaging of Ou4}

The imaging was carried out on 18 August 2012 at the F/3.3 $2.5 \mathrm{~m}$ Isaac Newton Telescope (INT) at the Observatorio del Roque de los Muchachos (ORM), La Palma, Spain. Images were taken with the Wide Field Camera (WFC) in three filters: a narrow-band [O III] filter with a central wavelength of $5008 \AA$ and a bandpass of $100 \AA$; a narrow-band $\mathrm{H} \alpha$ filter with a central wavelength of $6568 \AA$ and a bandpass of $95 \AA$, thus including the [N II] doublet around $\mathrm{H} \alpha$; and a broadband Sloan $g$ filter, with a central wavelength of $4846 \AA$ and a bandpass of $1285 \AA$. The plate scale is $0 . \mathrm{pix}^{-1}$, and the seeing varied from $1^{\prime \prime} .2$ to $2^{\prime \prime} .0$ during the night. We adopted six different telescope offsets that generously overlapped to cover the totality of Ou4 and the gaps between CCDs in the detector mosaic because the field of view of the WFC is $34 \times 34 \mathrm{arcmin}^{2}$. At each of them, we exposed for a total of between $15 \mathrm{~min}$ and $45 \mathrm{~min}$ in [O III], 5 and $15 \mathrm{~min}$ in $\mathrm{H} \alpha$, and $90 \mathrm{sec}$ in the $g$ filter. The night was dark: any significant moonlight would prevent detection of the nebula over the background given its very low surface brightness (see Sect. 4).

Images were reduced using the INT+WFC pipeline in Cambridge (CASU). The precise astrometric solution allowed a careful combination of all images using the swarp software (Bertin et al. 2002). The final mosaic has a field of view of roughly $0.9 \times 1$. 4 . Because $\mathrm{Ou} 4$ and the surrounding $\mathrm{H}$ II region Sh 2-129 cover the whole field of view of the camera, accurate background subtraction proved to be difficult and had to be carefully tuned. Figure 4 shows the colour-composite INT image of $\mathrm{Ou} 4$, while Fig. 5 shows the $[\mathrm{O} \mathrm{III}]$ and $\mathrm{H} \alpha+[\mathrm{N}$ II $]$ images with labels on the most relevant morphological features discussed below.

\subsection{Spectroscopy of the lobe tips}

Spectra of Ou4 were obtained on 17 August 2012 with the $4.2 \mathrm{~m}$ William Hershel Telescope (WHT) and the double-arm ISIS spectrograph. The long slit of ISIS was opened to $1^{\prime \prime}$-width and positioned at the southern tip of the nebula at PA $=344^{\circ}$, crossing the bright ridge of the nebula at coordinates RA = $21^{\mathrm{h}} 13^{\mathrm{m}} 23^{\mathrm{s}} .8$ and Dec $=+59^{\circ} 23^{\prime} 40^{\prime} .5(\mathrm{~J} 2000.0)$, as indicated in Fig. 6 by the long blue slit.

In the blue arm of ISIS, grating R300B was used, providing a dispersion of $1.7 \AA$ per (binned $\times 2$ ) pixel, a resolution of $3.5 \AA$, and a spectral coverage from 3600 to $5200 \AA$. In the red arm, grating R158R gave a dispersion of $1.84 \AA$ pix $^{-1}$, a resolution

7 For the $\mathrm{Aa}, \mathrm{Ab}$, and $\mathrm{B}$ components, the masses are 17.8, 14.2, and $16.7 M_{\odot}$; the effective temperatures are 31500,29100 , and $30800 \mathrm{~K}$ (corresponding to B0 V, B0.5 V, and B0 V spectral types according to Pecaut \& Mamajek 2013; the luminosities are 41370, 20398, and $33595 L_{\odot}$; the intrinsic $(B-V)_{0}$ colours are $-0.279,-0.265$, and -0.275 ; the observed $V$-band magnitudes are $6.579,7.179$, and 6.757 mag. 


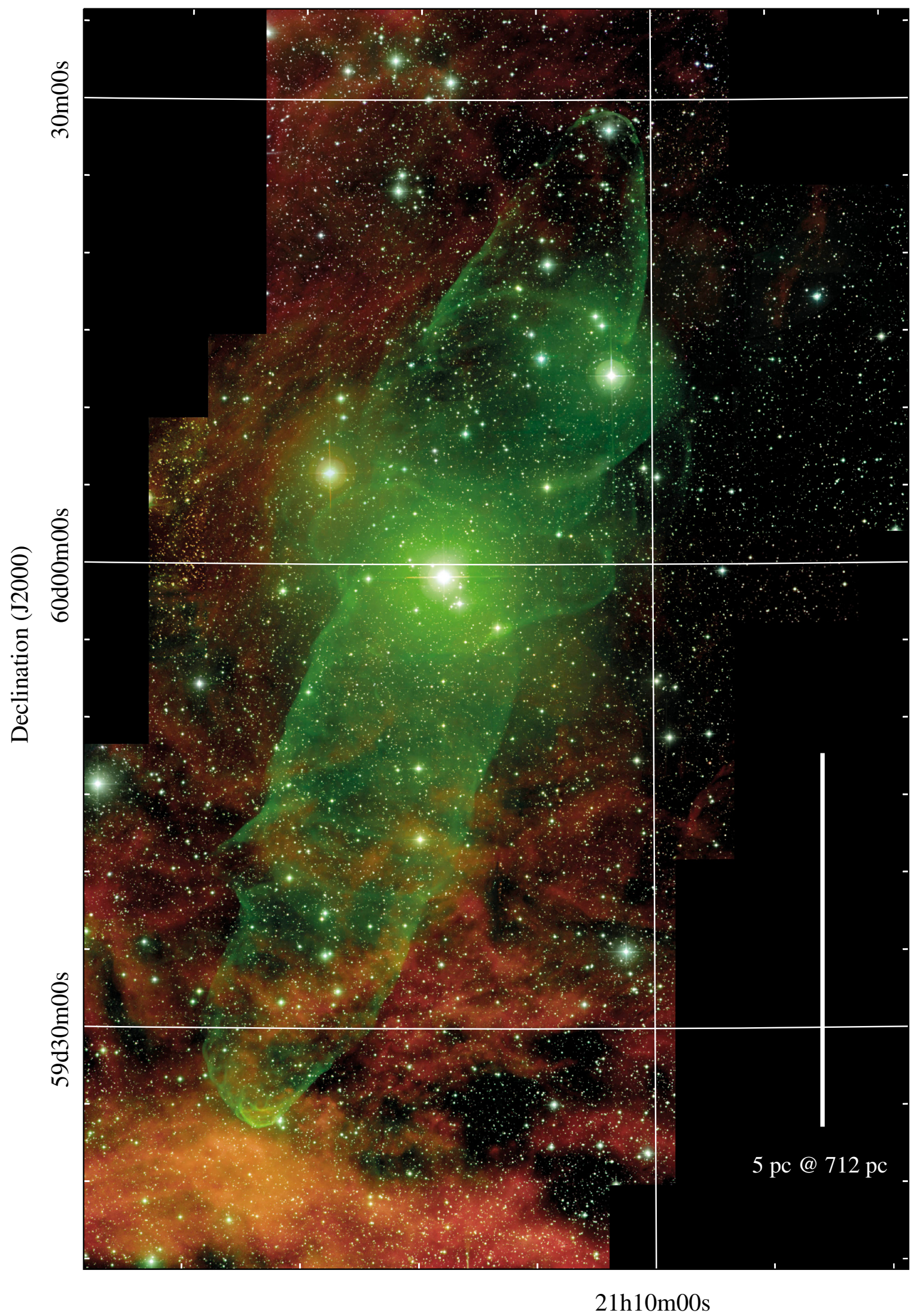

Right Ascension (J2000)

Fig. 4. Colour-composite mosaic image of Ou4 obtained with the Wide Field Camera of the $2.5 \mathrm{~m}$ INT. The [O III] 5007 emission is mapped in green, $\mathrm{H} \alpha+[\mathrm{N} \mathrm{III}]$ in red, and the broadband $g$ filter in blue. The intensity scale is logarithmic. The field of view is $1.36 \times 0.89$. The vertical line indicates the linear size if Ou4 is at the adopted distance of HR 8119 and Sh 2-129 (712 pc). North is up, east is left. 


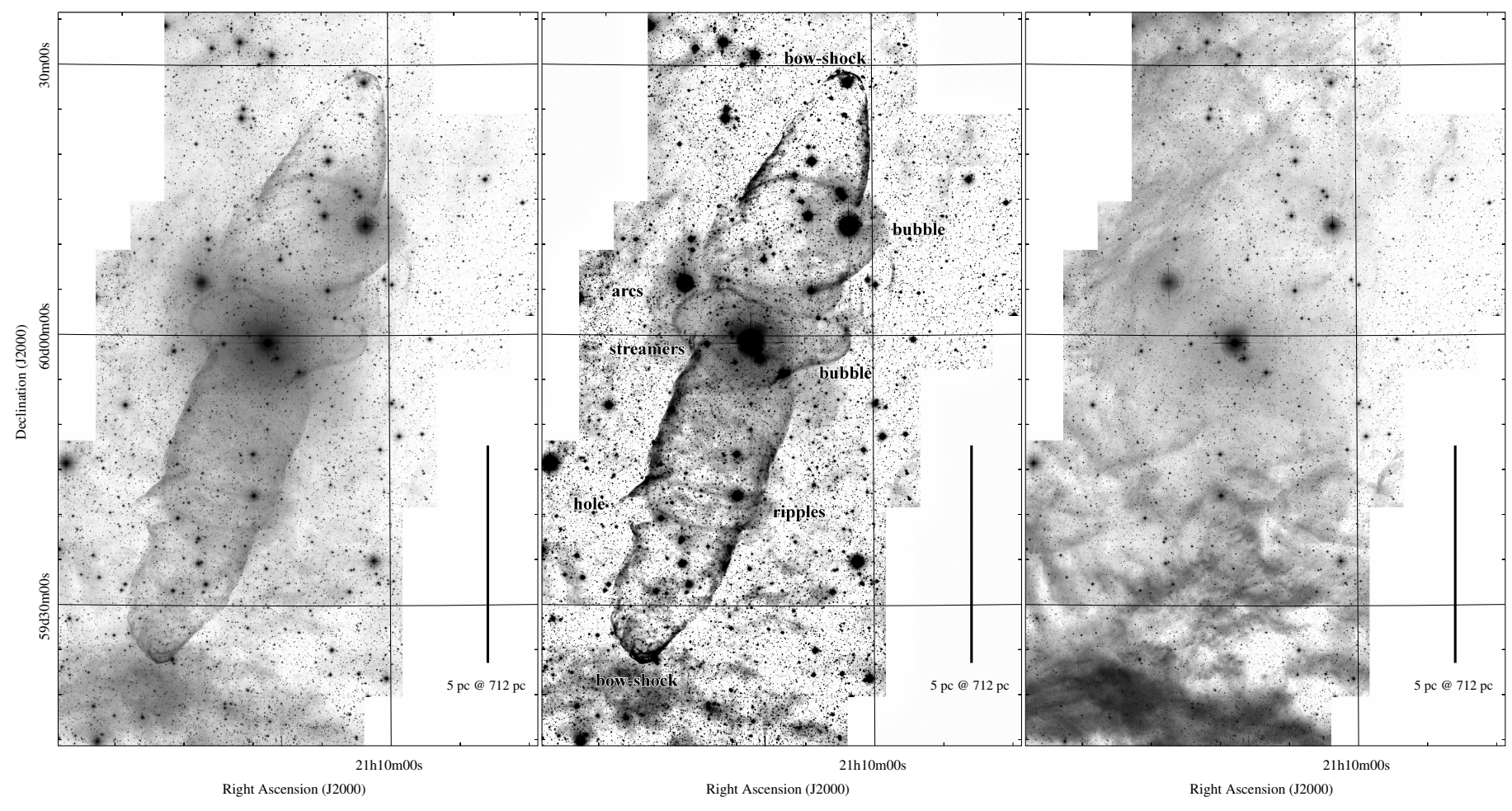

Fig. 5. [O III] 5007 (left and middle) and $\mathrm{H} \alpha+[\mathrm{N} \mathrm{II}]$ (right) mosaic images of Ou4. The sharpness of the [O III] 5007 image was enhanced in the middle panel using a blurred mask. The colour scale is logarithmic in the left and right panels. The field of view in each panel is $1.36 \times 0.89$. North is up, east is left.

of $3.5 \AA$, and a spectral coverage from 5400 to $7800 \AA$. The total exposure times was two hours. The spatial scale was 0.4 per binned pixel in the blue, and 0. '44 in the red. Seeing was $11^{\prime \prime} 0$. The spectrophotometric standard BD+28 4211 from Oke (1990) was observed during the night for flux calibration.

To determine the basic kinematic properties of the outflow, spectra at a higher resolution were also obtained on 24 October and 25 December 2012 using the same instrument, but grating 1200B. Useful data are limited to the [O III] $5007 \AA$ line, at which a spectral dispersion of $0.22 \AA$ pix $^{-1}$ and a resolution of $0.84 \AA$ was achieved with the adopted 0 ' $^{\prime} 9$ slit width. The slits, also indicated in Fig. 6 in red, cross the south and north tips of the lobes of Ou 4 at positions $21^{\mathrm{h}} 13^{\mathrm{m}} 25^{\mathrm{s}} .2,+59^{\circ} 23^{\prime} 46^{\prime \prime} .7(\mathrm{~J} 2000)$ at $\mathrm{PA}=340^{\circ}$, and $21^{\mathrm{h}} 10^{\mathrm{m}} 21^{\mathrm{s}} .8,+60^{\circ} 29^{\prime} 10^{\prime} .^{\prime} 6$ at PA $=20^{\circ}$, respectively. Total exposure times were $40 \mathrm{~min}$ in the south lobe, and $80 \mathrm{~min}$ in the fainter north lobe.

Spectra were reduced with the standard procedure using the longslit package of iraf $V 2.16^{8}$.

\section{Analysis}

\subsection{Overall morphology of Ou4}

The bipolar nebula is mainly visible in the [O III] 5007 light (Fig. 4, and left and middle panels of Fig. 5), whose surface brightness ranges from a few $10^{-16} \mathrm{erg} \mathrm{cm}^{-2} \mathrm{~s}^{-1} \operatorname{arcsec}^{-2}$ (tip of southern lobe, see Sect. 4.3) down to the detection limit of our images of several $10^{-17} \mathrm{erg} \mathrm{cm}^{-2} \mathrm{~s}^{-1} \operatorname{arcsec}^{-2}$ (in regions not contaminated by the diffraction halo of bright stars).

\footnotetext{
8 Iraf is distributed by the National Optical Astronomy Observatory, which is operated by the Association of Universities for Research in Astronomy (AURA) under cooperative agreement with the National Science Foundation.
}

As described in Acker et al. (2012), Ou4 is mainly composed of two collimated lobes with arc-shaped tips of enhanced [O III] emission. Their extremities recall the bow-shocks observed in collimated stellar outflows such as Herbig-Haro objects in the outflows from young stellar objects (Reipurth \& Bally 2001), or in high-velocity bipolar PNe. The south lobe, of cylindrical shape, is longer than the northern one, which instead has a more conical overall morphology. Neither lobe can be easily followed back to the central region, where a large distorted bubble is visible. This prevents an unambiguous association of the nebula with one of the stars near its symmetry centre. Only the eastern ridge of the south lobe extends far enough back to provide some useful indication. The lobe is wide until near the centre, where its edge is bowl-shaped and bends in a direction approximately pointing towards HR 8119 (Fig. 7). However, detection of the faint emission from the lobe at distances smaller than $\sim 2.2^{\prime}$ from HR 8119 is prevented by the bright diffraction halo of this star. This marked lobe curvature near the centre is similar to that of the inner lobes of the bipolar PN Mz 3 (Santander-García et al. 2004), for instance, or of several proto-PN such as Hen 401 (Sahai et al. 1999; Balick et al., in prep.). The overall morphology of Ou4 is very similar to that of the giant bipolar PN KjPn 8 (López et al. 1995), which has a size of $7.3 \mathrm{pc} \times 2.1 \mathrm{pc}$ for the kinematically determined distance of $1.8 \pm 0.3 \mathrm{kpc}$ (Boumis \& Meaburn 2013).

Compared with the discovery image, the INT mosaic reveals a wealth of new details. In addition to the central bubble, another elliptical bubble breaks the inner regions of the northern lobe. Moreover, in the central region of the south lobe a hole is visible as well as ripples directed perpendicularly to its longaxis. East of the central distorted bubble, additional features in the form of streamers and arcs can be identified (Fig. 5). Finally, the tips of the lobes, and in particular the southern one, are 
Table 1. Main properties of Ou4.

\begin{tabular}{|c|c|c|}
\hline \multicolumn{3}{|c|}{ Ou4 } \\
\hline $\begin{array}{l}\text { Lobe angular size } \\
\text { Linear size } \\
\text { Centre* } \\
\text { HR } 8119\end{array}$ & $\begin{array}{l}1: 16 \times 0.20 \\
14.4 \mathrm{pc} \times 2.5 \mathrm{pc} \\
21^{\mathrm{h}} 11^{\mathrm{m}} 45.6+59^{\circ} 59^{\prime} 00^{\prime \prime} \\
21^{\mathrm{h}} 11^{\mathrm{m}} 48.2+59^{\circ} 59^{\prime} 11^{\prime \prime} 8 \\
98^{\circ} .5202 \quad+07^{\circ} .9852\end{array}$ & $\begin{array}{l}\text { (712 pc) } \\
\text { Equ. J2000 } \\
\text { Equ. J2000 } \\
\text { Gal. J2000 }\end{array}$ \\
\hline \multicolumn{3}{|c|}{ Tip of the south lobe } \\
\hline $\begin{array}{l}\text { Optical extinction } \\
T_{\mathrm{e}}([\mathrm{O} \mathrm{III}],[\mathrm{O} \mathrm{II}]) \\
N_{\mathrm{e}}([\mathrm{S} \mathrm{II}]) \\
\text { Proper motion } \\
\text { RV range }^{\dagger} \\
\text { Velocity }^{-}\end{array}$ & $\begin{array}{l}1.1 \pm 0.4 \mathrm{mag} \\
55000 \mathrm{~K}, \geq 20000 \mathrm{~K} \\
\geq 50-100 \mathrm{~cm}^{-3} \\
\lesssim 00^{\prime} 06 \pm 00^{\prime} 03 \mathrm{yr}^{-1} \text { tentative } \\
-100-+10 \mathrm{~km} \mathrm{~s}^{-1} \\
112 \mathrm{~km} \mathrm{~s}^{-1}\end{array}$ & $\begin{array}{l}\text { See Sect. } 4.3 \\
\text { See Sect. } 4.3 \\
\text { See Sect. } 4.4 .2 \\
\text { See Sect. } 4.4 .3\end{array}$ \\
\hline \multicolumn{3}{|c|}{ Tip of the north lobe } \\
\hline $\begin{array}{l}\mathrm{RV}_{\text {range }}^{\dagger} \\
\text { Velocity }\end{array}$ & $\begin{array}{l}-55-+40 \mathrm{~km} \mathrm{~s}^{-1} \\
83 \mathrm{~km} \mathrm{~s}^{-1}\end{array}$ & See Sect. 4.4 .3 \\
\hline
\end{tabular}

Notes. ${ }^{*}$ Approximate symmetry centre of the Ou4 nebula. ${ }^{(\dagger)}$ Heliocentric, determined in the region covered by the slit (see Fig. 9).
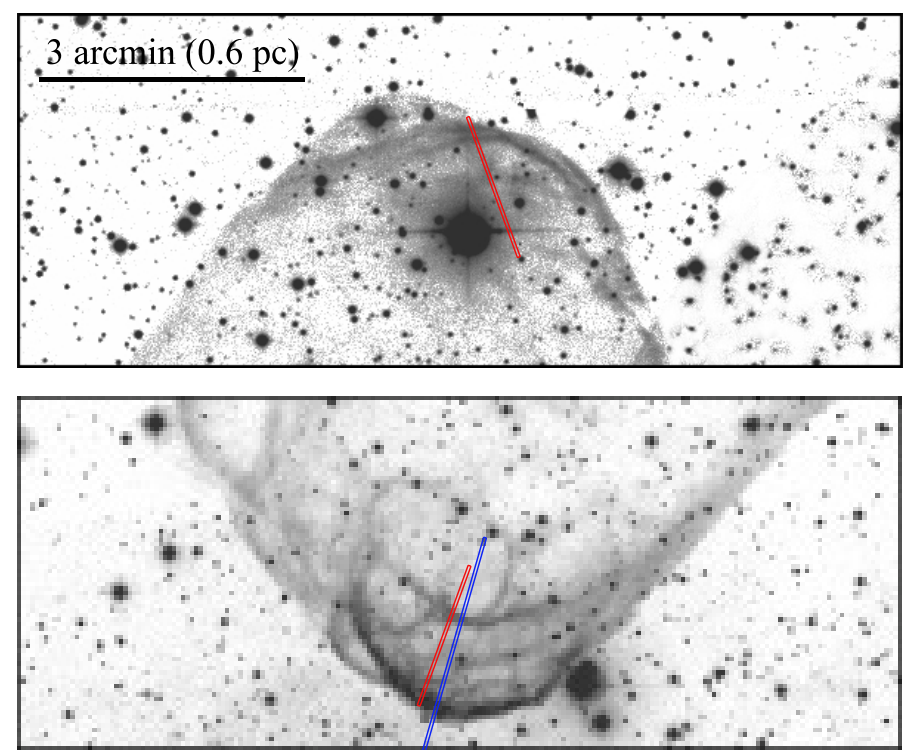

Fig. 6. Details of the north (top) and south (bottom) bow-shocks in the [O III] light. The field of view in each panel is $10^{\prime} \times 4^{\prime}$. North is up, east is left. The slit position for the lower resolution spectrum is indicated by the long (blue) slit. The reference star 2MASS J21131972+5925365 adopted as the zero-point of the $x$-axis in Fig. 8 is visible at its north end. The positions for the higher resolution spectra are indicated by the short (red) slits.

composed of complex systems of multiple arcs (see also Fig. 6). The main properties of Ou4 determined in this work are summarised in Table 1.

In the $\mathrm{H} \alpha+[\mathrm{N}$ II $]$ filter (Fig. 4, and right panel of Fig. 5), emission is dominated by ambient gas belonging to the H II region Sh 2-129 with little contribution from Ou4 except at specific regions like the south and north bow-shocks.

\subsection{Emission-line distribution in the tip of the south lobe}

Insights into the excitation and dynamics of the nebula are gained from examining the spatial distribution of different ions. This is shown in Fig. 8 for the lower resolution ISIS spectrum

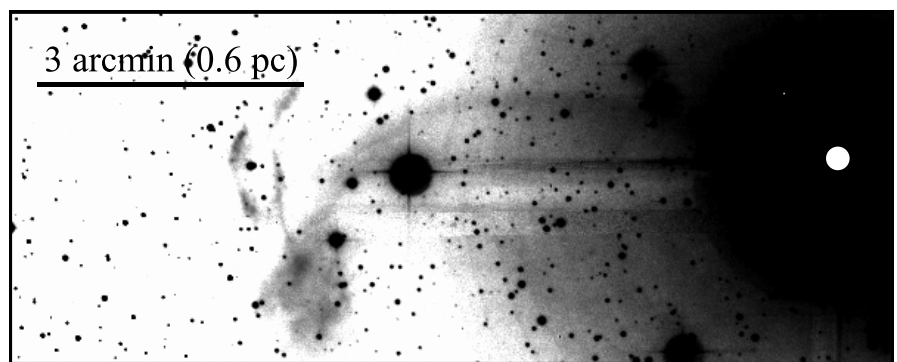

Fig. 7. Innermost region of the south lobe in the [O III] light. The field of view is $10^{\prime} \times 4^{\prime}$. North is up, east is left. Its eastern edge has a marked curvature and approximately points toward HR 8119 (indicated by the white circle).

that shows a cut of the tip of the southern lobe approximately south-north (cf. bottom panel of Fig. 6). The upper panel of Fig. 8 shows the spatial distribution of representative lines with the highest ionisation potential, such as [O III] 5007 and [Ne III] 3869, while the middle and bottom panels present those with lower ionisation potential ( $\mathrm{H} \alpha,[\mathrm{N} \mathrm{II}],[\mathrm{S} \mathrm{II}]$, and [O II]). Profiles were smoothed with a boxcar filter size of 3 spatial pixels (1".3). The field star 2MASS J21131972+5925365, visible at the upper end of the long blue slit in the bottom panel of Fig. 6, was used to anchor the blue and red spectra and define the zeropoint of the spatial scale. Negative distances refer to positions south of the reference star.

The sharp edge of the southern lobe corresponds to the peak in the [O III] 5007 emission at a distance $d=-115^{\prime \prime}$. [Ne III] has a similar behaviour, although the main peak is not equally pronounced. Ahead of the [O III] peak (i.e., at $d<-115^{\prime \prime}$ ), significant $\mathrm{H} \alpha$ and [N II], and to less extent [O II] and [S II] emission, can be associated with the HII region Sh 2-129. These lower ionisation ions also contribute to the emission of Ou4, as shown by the several peaks at $d>-115^{\prime \prime}$, but their overall distribution is obviously shifted to the north (i.e., inside the lobe) compared with [O III]. In particular, [O II] 3727, which is at least as bright as [O III] 5007 (even taking into account mixing with the emission of Sh 2-129), peaks about two arcseconds north of [O III]. Some faint [O I] 6300 emission, not shown in Fig. 8, is also detected inside the lobe. No significant differences are seen in the profiles of other lines not shown in Fig. 8, and in particular [O III] 4363 is similar to [O III] 5007, $\mathrm{H} \beta$ to $\mathrm{H} \alpha$, and [S II] 6731 to [S II] 6716.

This overall distribution, with the higher ionisation ions outward and the lower ionisation ions progressively inward, is a first indication that the gas in the tip of the south lobe of Ou4 is shock-excited. Photoionisation from a central source would instead produce an opposite stratification with the higher ionisation ions inwards.

\subsection{Gas physical conditions from emission-line ratios}

Line fluxes for the Ou4 outflow were measured in the lowresolution spectrum that crosses the tip of the south lobe. A precise background subtraction is limited by the overlapping emission of the HII region Sh 2-129, whose surface brightness is highly variable at different spatial scales, as can be seen in the right panel of Fig. 5 and in Fig. 4. We performed several tests and finally adopted a background (night sky + Sh 2-129) determined using the portion of the spectrum of the northernmost part of the slit, near the adopted reference star (Fig. 6). This is likely to leave some small contamination by Sh $2-129$. However, the main 
R. L. M. Corradi et al.: Gas physical conditions and kinematic of the giant outflow Ou4

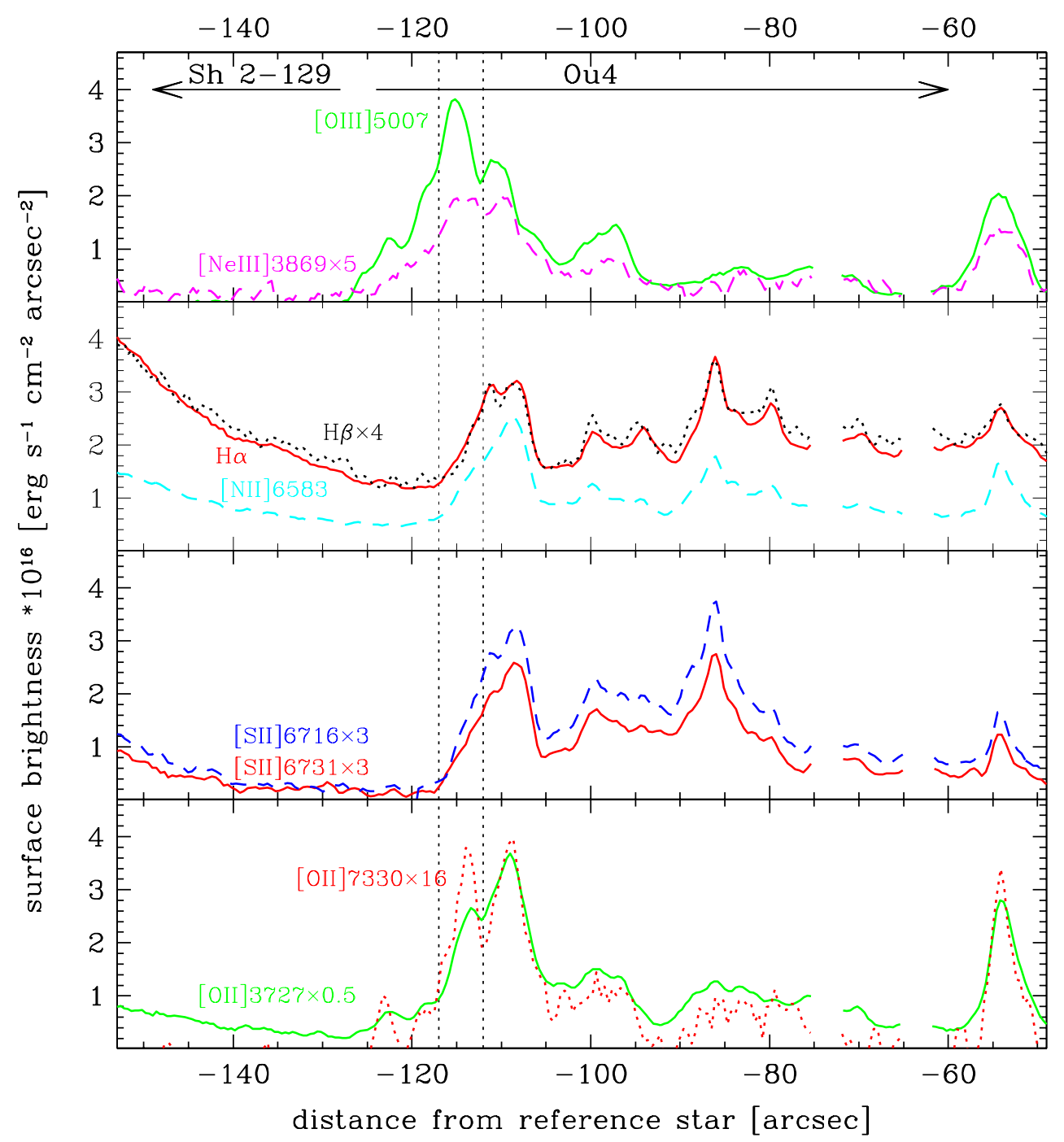

Fig. 8. Spatial profile of selected emission lines through the tip of the southern lobe, along the slit shown in blue in Fig 6. Emission of different ions has been scaled by the factors indicated in the labels. Regions corresponding to field stars are masked. The vertical dotted line shows the integration limits for the line flux measurements presented in Table 2.

conclusions of the analysis presented below are not affected by a different choice of the region used for background subtraction.

The ionisation stratification discussed in the previous section makes the line ratios highly dependent on the selected spatial region over which the flux is integrated. We present in Table 2 line fluxes measured in the shocked gas between $-117^{\prime \prime}$ and $-112^{\prime \prime}$ from the reference star in the south lobe, around the main peak of the [O III] emission. These integration limits are indicated by the vertical dotted lines in Fig. 8 and do not include the cooling region of lower excitation behind the shock.

To derive physical quantities from these fluxes, the nebular extinction is required. The logarithmic extinction constant $c_{\beta}$ was determined from the Balmer decrement by averaging the results from the observed $\mathrm{H} \alpha / \mathrm{H} \beta$ and $\mathrm{H} \gamma / \mathrm{H} \beta$ ratios in our long-slit spectra. The theoretical Balmer line ratios were adopted from Brocklehurst (1971) for electron temperatures between 10000 and $20000 \mathrm{~K}$ and a low-density regime $\left(N_{\mathrm{e}} \sim 10^{2}\right.$, see below). We did not use only the narrow spatial region of the shocked gas around the [O III] peak to determine an average value of the extinction of the south lobe of Ou4 (which can be patchy at these relatively large angular sizes). Instead, we considered a broader area between $-117^{\prime \prime}$ and $-105^{\prime \prime}$ from the reference star, where most of the Balmer line emission in the region covered by our long slit is produced (see Fig. 8). This also includes part of the cooling region behind the shock. We derive a value of $c_{\beta}=0.5 \pm 0.2$ using the reddening law of Fitzpatrick (1999) and $R_{V}=3.1$. This corresponds to $A_{V}=1.1 \pm 0.4 \mathrm{mag}$. The same value, within the errors, is found for the emission from Sh 2-129 ahead of the shock. This $c_{\beta}$ value was adopted to deredden the fluxes in Table 2. This foreground-extinction value is also similar to the value that we found for HR 8119 in Sect. 2.

Physical conditions in the gas (electron density and temperature) were then computed using the nebular package within iraf, based on the five-level atom program originally published by De Robertis et al. (1987), and further developed by Shaw \& Dufour (1995). From the [S II] doublet, we obtain an electron density $N_{\mathrm{e}}$ of between $50 \mathrm{~cm}^{-3}$ and $100 \mathrm{~cm}^{-3}$ depending on the adopted temperature. This is a lower limit for the gas density in the tip of the south lobe of Ou4, as the [S II] emission mainly comes from the inside boundary of the selected region of the spectrum (see Fig. 8) and therefore traces a different nebular zone than [O III]. In addition, non-negligible residual contribution of [S II] emission from the overlapping lowdensity Sh 2-129 could be present. The electron temperature $T_{\mathrm{e}}$ 
Table 2. Observed and dereddened (adopting $c_{\beta}=0.5$ ) line fluxes in the shock region between -112 and $-117^{\prime \prime}$ (see Fig. 8) of the south lobe.

\begin{tabular}{|c|c|c|}
\hline \multirow[t]{2}{*}{ Line identification } & \multicolumn{2}{|c|}{ Flux } \\
\hline & Observed & Dereddened \\
\hline [O II] $3726+3729$ & 883.1 & 1262.3 \\
\hline [Ne III] 3869 & 72.5 & 99.3 \\
\hline$[\mathrm{Ne}$ III $] 3968+\mathrm{H} \epsilon$ & 35.9 & 47.7 \\
\hline $\mathrm{H} \delta 4101$ & 18.9 & 24.1 \\
\hline $\mathrm{H} \gamma 4340$ & 45.7 & 53.8 \\
\hline [O III] 4363 & 55.4 & 64.7 \\
\hline $\mathrm{H} \beta 4861$ & 100.0 & 100.0 \\
\hline [O III] 4959 & 237.8 & 231.1 \\
\hline [O III] 5007 & 717.5 & 687.5 \\
\hline [N II] 6548 & 94.3 & 63.1 \\
\hline $\mathrm{H} \alpha 6563$ & 503.7 & 336.1 \\
\hline [N II] 6583 & 308.3 & 205.0 \\
\hline [S II] 6716 & 114.4 & 74.2 \\
\hline [S II] 6731 & 87.0 & 56.3 \\
\hline [O II] 7319 & 51.8 & 30.6 \\
\hline [O II $] 7330$ & 53.7 & 31.7 \\
\hline
\end{tabular}

Notes. Fluxes are normalised to $\mathrm{H} \beta=100$. The observed $\mathrm{H} \beta$ integrated flux in the area is $4.03 \times 10^{-16} \mathrm{erg} \mathrm{cm}^{-2} \mathrm{~s}^{-1}$. The derived physical quantities are $N_{\mathrm{e}}([\mathrm{S} \mathrm{II}])=50-100 \mathrm{~cm}^{-3}$ and $T_{\mathrm{e}}([\mathrm{O} \mathrm{III}])=55000 \mathrm{~K}$.

derived from the $[\mathrm{O} \mathrm{III}](5007+4959) / 4363$ line ratio is as high as $55000 \mathrm{~K}$ for this density. This is a very high $T_{\mathrm{e}}$, which could be reduced to values typical of photoionised nebulae only if $N_{\mathrm{e}}$ had the value of $10^{7} \mathrm{~cm}^{-3}$ in the $\mathrm{O}^{2+}$ emitting region. On the other hand, [O II] is expected to be produced in the same region as [S II] (but note the additional emission peak of [O II]7330 in Fig. 8), and therefore the [S II] density can be adopted to compute $T_{\mathrm{e}}$ from the $[\mathrm{O}$ II $](3726+3729) /(7320+7330)$ line ratio. The latter ratio also suggests a high electron temperatures $(\geq 20000 \mathrm{~K})$, even though at low densities the [O II] indicator reaches its asymptotic value.

This $T_{\mathrm{e}}$, too high to be produced by photoionisation, is another proof that the gas at the tip of the lobes of Ou4 is ionised by shocks. This conclusion is also supported by standard shock-excitation diagnostic line ratios. In this nebular region, $\log (\mathrm{H} \alpha /[\mathrm{N}$ II $] 6548+6583)=0.1$ and $\log (\mathrm{H} \alpha /[\mathrm{S} \mathrm{II}] 6716+6731)=0.4$, which locates Ou4 together with shock-excited sources such as Herbig-Haro objects (see e.g. Viironen et al. 2009). The same conclusion is reached by analysing other portions of the slit. The lower-excitation emission inside the lobes is therefore expected to be the recombining/cooling region behind the shock. By integrating the $\left[\mathrm{N}\right.$ II] $(6548+6583) / 5755$ between slit positions $-117^{\prime \prime}$ and $-105^{\prime \prime}$, including the main [N II] emission peak, we consistently obtain a lower temperature, $T_{\mathrm{e}} \sim 14400 \mathrm{~K}$.

The electron density of gas in the relatively bright region of Sh 2-129 ahead of the bow-shock (from $-150^{\prime \prime}$ to $-130^{\prime \prime}$ from reference star), determined from the [S II]67166731 doublet, is $N_{\mathrm{e}}=55 \mathrm{~cm}^{-3}$, assuming a typical $T_{\mathrm{e}}$ for H II regions of $10000 \mathrm{~K}$. Because this emission region is located on the ionised boundary of the $\mathrm{H}$ II region, this electronic density is that of the molecular cloud.

To estimate the electronic density inside the $\mathrm{H}$ II region we used the $\mathrm{H} \alpha$ emission measure along the line of sight, $\mathrm{EM}=$ $\int_{\mathrm{H}} N_{\mathrm{e}}^{2} \mathrm{~d} s$, which can be computed from the $\mathrm{H} \alpha$ surface brightness corrected from extinction, $I$, with the following formula: $\mathrm{EM} /\left(1 \mathrm{~cm}^{-6} \mathrm{pc}\right)=2.75 \times(T / 10000 \mathrm{~K})^{0.9} \times(I / 1 R)$, which is valid for temperatures between 5000 and $10000 \mathrm{~K}$, and where $R$ is one Rayleigh, i.e., $2.42 \times 10^{-7}$ ergs $\mathrm{cm}^{-2} \mathrm{~s}^{-1} \mathrm{sr}^{-1}$ at the $\mathrm{H} \alpha$ wavelength (Reynolds 1977). We assumed for the H II region a typical temperature of $10000 \mathrm{~K}$. From the Virginia Tech Spectral line Survey (VTSS; Finkbeiner 2003), which mapped the north-west half-part of Sh 2-129 in H $\alpha$ with 6' (FWHM) resolution, we obtained the radial profile from HR 8119 for a position angle of $\approx 26^{\circ}$ of the $\mathrm{H} \alpha$ intensity in $R$ unit, and dereddened it using $A_{V}=1.1 \mathrm{mag}$ and the extinction law of Fitzpatrick (1999). This intensity is modeled with a uniform intensity of $22.3 R$ plus a constant-density (hemi-)spherical shell geometry to reproduce the limb brightening (e.g., Nazé et al. 2002), and convolved with a 6 '-FWHM Gaussian. To reproduce the external profile we need a shell-radius of 0.76 (i.e., 9.4 pc at a distance of $712 \mathrm{pc}$ ), a shell-thickness of 0.14 , and a shell-density of $5.1 \mathrm{~cm}^{-3}$. The constant-density inside the shell is estimated to be about $1.7 \mathrm{~cm}^{-3}$. This lower electronic density of the blister H II region compared with that of the molecular cloud is the result of the ionisation and heating by the central early-type B stars, which have produced the Champagne flow.

If Ou4 is embedded in Sh 2-129, the pre-shock gas density is the electronic density inside this H II region. Since this shock is radiative, the gas behind the shock relaxes to the H II region temperature, and if there is no magnetic field the shock-compression ratio scales as Mach-number squared (Draine \& McKee 1993). Taking $\gamma=5 / 3$ for the ratio of specific heat and $\mu=0.7$ for the mean molecular weight inside the H II region, the sound velocity is $14 \mathrm{~km} \mathrm{~s}^{-1}$, and a shock velocity of $112 \mathrm{~km} \mathrm{~s}^{-1}$ (see below Sect. 4.4.3) gives a Mach number of 8.0, which leads to a compression ratio of 64 . Therefore, the post-shock gas density is estimated to be $110 \mathrm{~cm}^{-3}$.

\subsection{Kinematics of the lobe tips}

\subsubsection{Radial velocities}

Heliocentric line-of-sight velocities in regions at the tip of each lobe were computed from the Doppler shift of the [O III] 5007 line in the higher resolution spectra described in Sect. 3.2. The slit locations are indicated in Fig. 6. Wherever possible, two Gaussians were fitted to the spectra at different slit positions, binning the information every 4 arcsec to increase the signal-to-noise ratio. The resulting position velocity plot is presented in Fig. 9. Accuracy in the double-Gaussian fitting is mainly limited by the separation and shape of the profiles of the two line components. At the positions where they cannot be resolved, only a single Gaussian could be measured with a generally high FWHM resulting from the blending of the two components. For this reason, we indicate in Fig. 9 the FWHM of the Gaussian line fits rather than the (smaller) formal error of the fitting.

The plot shows that there is only a small overall velocity difference between the two bow-shocks, indicating that the outflow is likely to be oriented near the plane of the sky. The north lobe would recede from us, and the south lobe approach us. Right inside each lobe, the [O III] emission can be split into two components that are separated by up to about $100 \mathrm{~km} \mathrm{~s}^{-1}$ in the small fraction of the lobe length covered by these observations. Therefore a lower limit for the lobe transverse velocity can be set to $50 \mathrm{~km} \mathrm{~s}^{-1}$.

In Fig. 9, the radial velocities of HR 8119 and Sh 2-129 are also shown. The velocity of the two lobes is symmetrically located with respect to the massive star and the H II region, which again supports the hypothesis that they might be associated with $\mathrm{Ou} 4$. 
R. L. M. Corradi et al.: Gas physical conditions and kinematic of the giant outflow Ou4

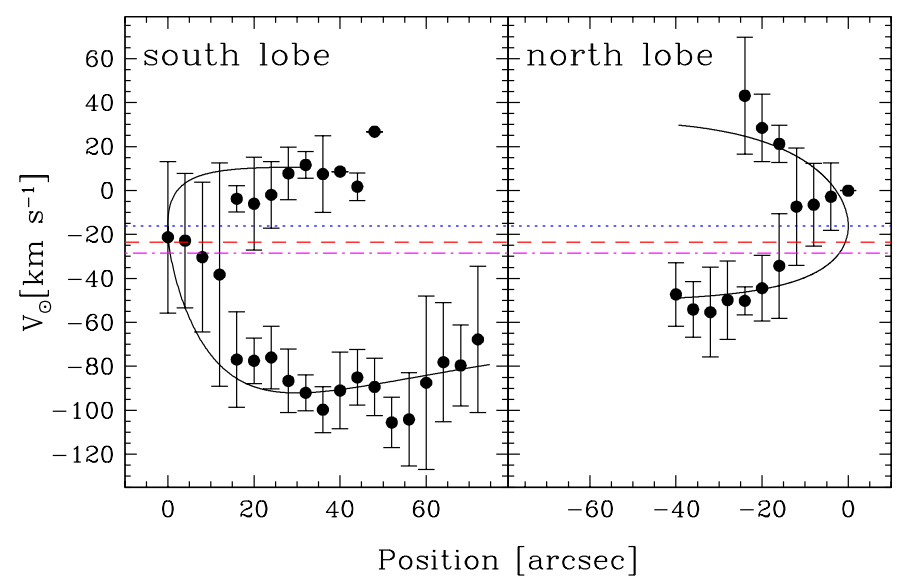

Fig. 9. Position-velocity plot at the tips of the lobes. Position zero is defined at the point at which the slit crosses the end of each lobe. Positive and negative positions indicate distances from this reference point along the slits and toward the centre of the nebulae in the south and north lobe. Error bars indicate the [O III] FWHM after correcting for the instrumental broadening. Solid lines show our bow-shock kinematic model (see Sect. 4.4.3). The horizontal blue dotted line indicates the heliocentric radial velocity of HR $8119\left(-16.2 \mathrm{~km} \mathrm{~s}^{-1}\right.$; Wilson 1953). The red dashed line is the $\mathrm{H} \alpha$ average velocity of Sh 2-129 obtained using Perot-Fabry measurements $\left(-23.5 \pm 5.3 \mathrm{~km} \mathrm{~s}^{-1}\right.$, Georgelin \& Georgelin 1970), while the dashed-dotted magenta line represents the $\mathrm{CO}$ peak located east of HR 8119 on the border of Sh 2-129 $\left(-28.5 \pm 0.7 \mathrm{~km} \mathrm{~s}^{-1}\right.$; Blitz et al. 1982).

\subsubsection{Proper motions}

We identified the tip of the south lobe in two $B$-band digitised plates obtained at two different epochs separated by nearly 41 years (Fig. 10). We used the imwcs software ${ }^{9}$ and the SExtractor software (Bertin \& Arnouts 1996) to match these $B$-band POSS1 and POSS2 images of Ou4 and our [O III] INT image to the 2MASS reference frame (Skrutskie et al. 2006).

However, the modest spatial resolution and the weak signalto-noise ratio prevent us from safely tracking any emission features between the POSS1 and POSS2 epochs. Our tentative pairing of a few emission features leads to a proper-motion upper limit of $\sim 0 .^{\prime} 06 \pm 0 .{ }^{\prime} 03 \mathrm{yr}^{-1}$ (or a tangential velocity of $\sim 200 \pm 100 \mathrm{~km} \mathrm{~s}^{-1}$ if Ou4 is located at the distance of HR 8119), where the proper-motion error includes the error of the image registration and the determination of the features position.

\subsubsection{Bow-shock kinematic model}

The edges of the brightest working-surfaces of the south- and north-lobe tips (Fig. 6) can be fitted with a parabola. Assuming axisymmetry (see Fig. 1 of Hartigan et al. 1987), we can then argue that the intrinsic shape of the working surface is a paraboloid, because the projection onto the plane of the sky of such a geometric figure for any inclination angle is a parabola (see appendix of Hartigan et al. 1990). The actual 3D shape can then be computed for a given inclination of the bow shock along the line of sight.

In the shock rest frame, the parallel component of the velocity of the incoming gas does not change throughout the working surface, whereas the perpendicular component of the velocity is strongly decreased (by a factor of 4 by the shock and about 10

\footnotetext{
9 The imwcs software is available at http://tdc-www.harvard. edu/wcstools/imwcs
}

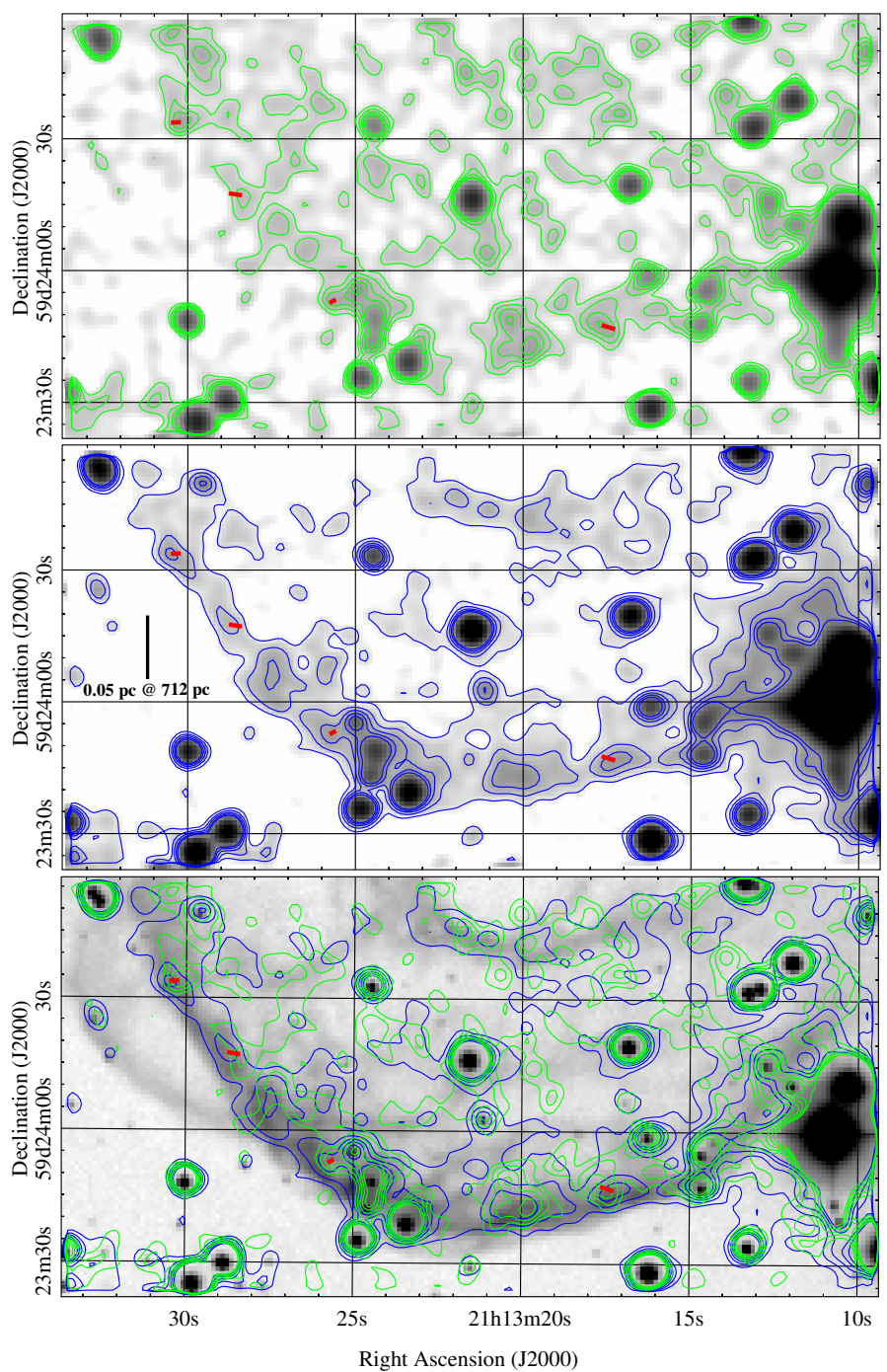

Fig. 10. Proper motions of the tip of the south lobe of Ou4. Top and middle panels: POSS1 (epoch 1952-07-22) and POSS2 (epoch 199306-25) blue plates in logarithmic greyscale smoothed with a Gaussian kernel $\left(\sigma=11^{\prime \prime} 5\right)$ with green and blue contours overlaid. Bottom panel: the green and blue contours overlaid on our [O III] INT mosaic (epoch 2012-08-19). The possible motions of four emission features between the POSS 1 and POSS 2 epochs are indicated in each panel by short red lines (average size of $\sim 22^{\prime \prime} 5 \pm 11^{\prime \prime} 1$ ).

by cooling; see Hartigan et al. 1987). Therefore, in the observer frame the velocity of the emitting post-shock material, which fills a rather thin shell behind the working surface, is nearly perpendicular to the paraboloid surface, its intensity is highest at the paraboloid vertex (i.e., equal to the shock velocity) and decreases from this position. The observed bow-shock size is directly controlled by the threshold value of the perpendicular component of the velocity that is required to produce the line emission conditions. For high inclinations the radial velocities derived by longslit spectroscopy have a characteristic hook shape in a velocityposition diagram, where the observed range of radial velocities is of the order of the actual bow-shock velocity (see Fig. 6 of Hartigan et al. 1990).

We built a toy model of this paraboloid bow-shock with the shape and size measured from our images where we can vary the systemic radial velocity, the bow-show velocity and inclination along the line of sight, and compute the resulting radial velocities of the emitting post-shock material along our long-slit 
positions. The systemic radial velocity is estimated by averaging the first four (unresolved) radial velocity measurements at the bow-shock heads, and is fixed to this value $\left(-16.2 \mathrm{~km} \mathrm{~s}^{-1}\right)$ in our simulations. We also assume the same minimum perpendicular component of the velocity of the incoming gas in the shock rest-frame. These simulated velocity-position diagrams are then compared with the observed gas kinematic.

We show in Fig. 9 our best match for the south and north bow-shocks, which was obtained for an inclination of $60^{\circ}$ and $100^{\circ}$ (where $0^{\circ}$ is a bow-shock directed towards us), and a shock velocity of 112 and $83 \mathrm{~km} \mathrm{~s}^{-1}$. The lower velocity of the north bow-shock may explain why it is fainter than the south bowshock. This toy models allows us to reproduce the global properties of the observed gas kinematics.

With these shock velocities the lowest value of the perpendicular component of the velocity of the incoming gas in the shock rest-frame is $55 \mathrm{~km} \mathrm{~s}^{-1}$. The lowest perpendicular velocity needed to observe [O III] is usually assumed to be equal to $90-100 \mathrm{~km} \mathrm{~s}^{-1}$, based on plane-parallel simulation of shock emission (e.g., Hartigan et al. 1987). However, if Ou4 is located inside the Sh 2-129 H II region, the pre-shocked gas already contains $\mathrm{O}^{+2}$ (see Fig. 1), and the perpendicular velocity needed to produce bright [O III] may be lower. Confirming this hypothesis would require detailed emission modelling of the bow-shock and the $\mathrm{H}$ II region, which is well beyond the scope of this article.

These south and north bow-shock velocities correspond to proper motions of $0{ }^{\prime} 028 \times$ and $0.024 \times(d / 712 \mathrm{pc})^{-1} \mathrm{yr}^{-1}$ and to kinematical time scales of $88300 \times$ and $87800 \times(d / 712 \mathrm{pc}) \mathrm{yr}$. The poorly constrained proper motion of the south bow-shock obtained in the previous section prevent us from deriving a secure kinematic distance to Ou4. However, any distance smaller than about $133 \mathrm{pc}$ can be excluded, because it would lead to an apparent expansion of the lobe tips larger than our proper-motion estimate plus three times our uncertainty.

\subsection{Central part of Ou4: [O III] emission vs. mid-infrared bubble}

\subsubsection{Morphology of the mid-infrared bubble}

Figure 11 shows a contour map of the mid-infrared bubble detected in the WISE W4-filter image in the central region of Ou4 overlaid on the [O III] image. The angular resolution of the W4-filter image is $12^{\prime \prime}$ (FWHM). The central point-like source is the mid-infrared counterpart of HR 8119 . The $5^{\prime}$-radius extended emission is asymmetric, with a surface brightness that is on average about three times higher on the eastern side than on the western side. There are two emission peaks, located $1.8^{\prime}$ east and $2.7^{\prime}$ north of HR 8119 . The north and south extensions of the mid-infrared bubble match the limit of the [O III] bubbles (see also the middle-left panel of Fig. 13 for the full range of the W4 intensity). A weak extension of the infrared bubble towards the east of HR 8119 corresponds to the base of the [O III] streamers. A ridge of mid-infrared emission is located at the south-west border of the mid-infrared bubble. Figure 12 is an enlargement of this region. The mid-infrared counterpart of the star HD 239597 (K2 spectral type in the Henry Draper catalogue and extension) is barely resolved from the ridge emission. This ridge emission corresponds to the [O III] filaments that defines the southern limit of the Ou4 bubble.

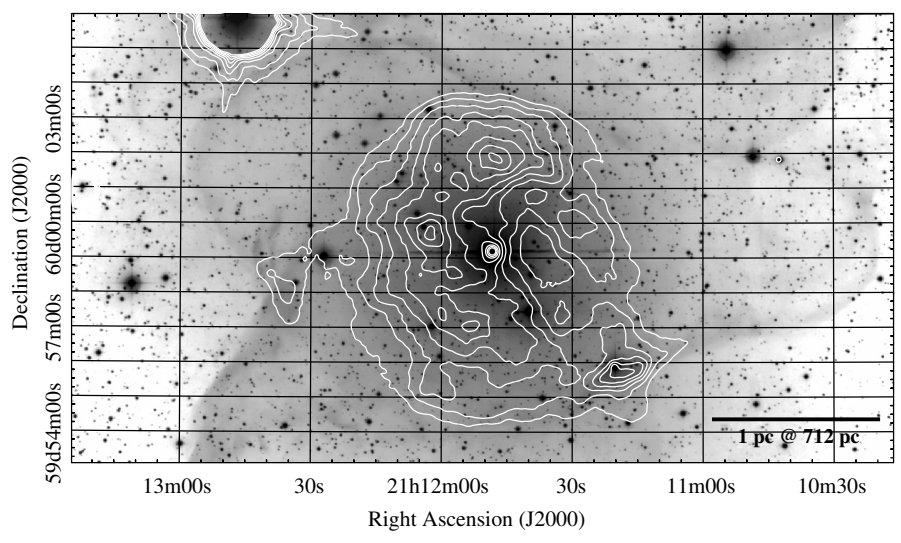

Fig. 11. [O III] emission from the central part of Ou4 vs. $22 \mu \mathrm{m}$ emission. The colour scale of the [O III] image is logarithmic. The contour map is the $22 \mu \mathrm{m}$ emission of the W4-filter image from 89.375 to 92.5 digital numbers (DN) with linear steps of $0.446 \mathrm{DN}$ (i.e., from 2.471 to $2.557 \mathrm{mJy} / \operatorname{arcsec}^{2}$ with linear steps of $\left.0.012 \mathrm{mJy} / \operatorname{arcsec}^{2}\right)$. The linear scale is shown in the bottom-right corner.

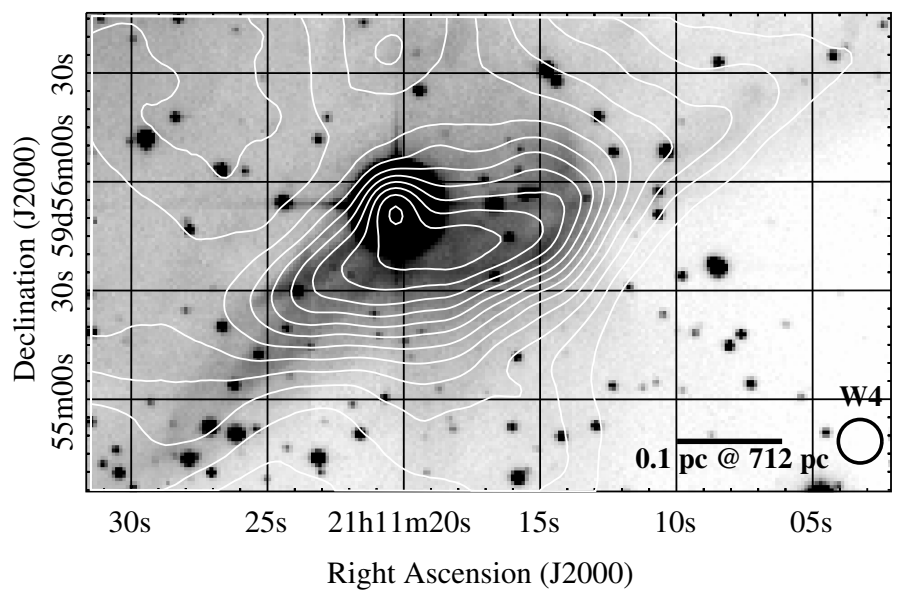

Fig. 12. Details of the [O III] emission of Ou4 near the ridge of midinfrared emission. The bright star is HD 239597. The colour scale of the [O III] image is linear. The contour map is the $22 \mu \mathrm{m}$ emission from the W4-filter image from 89.375 to 92.5 digital numbers (DN) with linear steps of $0.223 \mathrm{DN}$ (i.e., from 2.471 to $2.557 \mathrm{mJy} / \mathrm{arcsec}^{2}$ with linear steps of $0.006 \mathrm{mJy} / \mathrm{arcsec}^{2}$ ). The linear scale and the angular resolution of the W4-filter image (12"-FWHM) are shown in the bottom-right corner.

\subsubsection{Spectral energy distribution of the mid-infrared bubble}

The location of the mid-infrared bubble inside the [O III] emission may suggest line emission from hotter oxygen gas (e.g., [O IV] at $\lambda=25.87 \mu \mathrm{m}$ ) and not continuum emission from dust grains. To constrain the nature of the mid-infrared bubble we therefore built a spectral energy distribution (SED) of the brightest emission peak by combining WISE $\left(\lambda_{\mathrm{eff}}=11.56\right.$ and $22.09 \mu \mathrm{m})$ and IRAS $\left(\lambda_{\mathrm{eff}}=10.15,21.73,51.99\right.$, and $95.30 \mu \mathrm{m})$ photometry. We used the WISE All-Sky Atlas images (Cutri et al. 2012) and the Improved Reprocessing of the IRAS Survey (IRIS; Miville-Deschênes \& Lagache 2005), which provided an angular resolution of $6^{\prime \prime} .5,12^{\prime \prime}, 3.8^{\prime}, 3.8^{\prime}, 4.0^{\prime}$, and $4.3^{\prime}$ (FWHM) at 12, 22, 12, 25, 60, and $100 \mu \mathrm{m}$, respectively.

Despite the lower angular resolution of IRAS, the east side of the mid-infrared bubble is detected in the IRIS $25-\mu \mathrm{m}$ image (see the middle-right panel of Fig. 13). We used a custom IDL program to perform circular-aperture photometry using a procedure adapted from DAOPHOT. In all images, the source+background 
R. L. M. Corradi et al.: Gas physical conditions and kinematic of the giant outflow Ou4
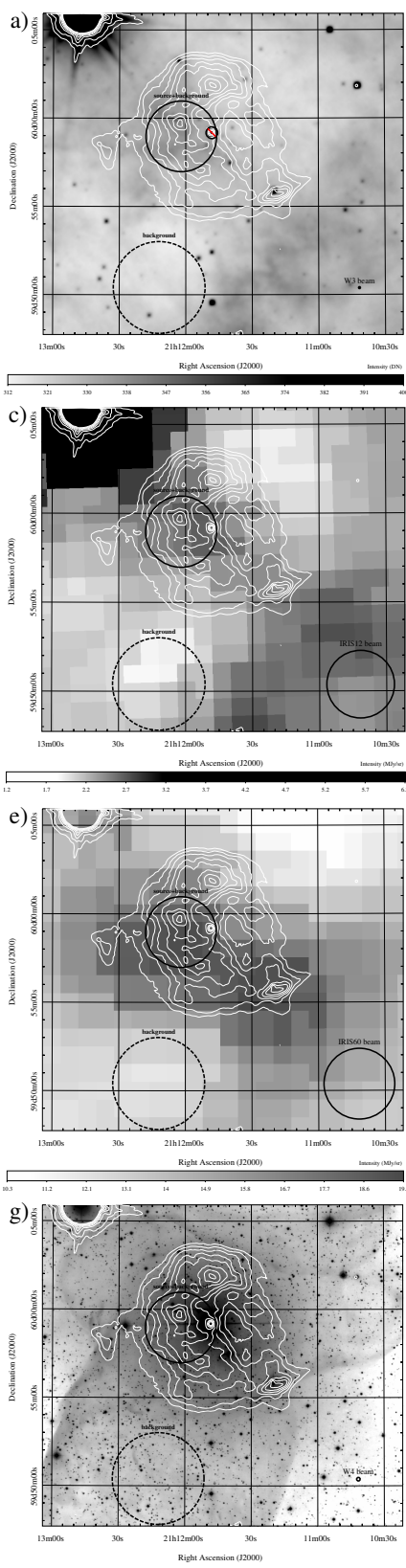

Fig. 13. Mid- to far-infrared views of the central part of Ou4. a) WISE $12 \mu \mathrm{m}$ (W3-filter) image. b) WISE $22 \mu \mathrm{m}$ (W4-filter) image. c) IRIS $12 \mu \mathrm{m}$ image. d) IRIS $25 \mathrm{~s} \mu \mathrm{m}$ image. e) IRIS $60 \mu \mathrm{m}$ image. f) IRIS $100 \mu \mathrm{m}$ image. g) INT [O III] image for comparison purpose. The contour map is the $22 \mu \mathrm{m}$ emission from 89.375 to 92.5 digital numbers (DN) with linear step of $0.446 \mathrm{DN}$ (i.e., from 2.471 to $2.557 \mathrm{mJy} / \operatorname{arcsec}^{2}$ with linear step of $0.012 \mathrm{mJy} / \operatorname{arcsec}^{2}$ ). The FWHM resolution is plot in the bottom-right corner of each panel. The two large circles are the regions used for the aperture photometry (see text and Fig. 14).

area is centred on $\left(21^{\mathrm{h}} 12^{\mathrm{m}} 02^{\prime \prime},+59^{\circ} 58^{\prime} 58^{\prime \prime}\right.$; J2000) with a radius of $2^{\prime}$ to match the angular resolution of IRIS images, and the background area is centred on $\left(21^{\mathrm{h}} 12^{\mathrm{m}} 12^{\prime \prime} \cdot 0,+59^{\circ} 50^{\prime} 24^{\prime \prime}\right.$; J2000) with a radius of 2!6 (Fig. 13). We subtract the resolved fluxes of HR 8119 in the WISE All-Sky catalog (Cutri et al. 2012) from the extended-source fluxes in the $W 3, W 4$, IRAS12, and IRASS25 bands.

The resulting SED (Fig. 14) shows significant flux emission in all bands, which is consistent with continuum emission

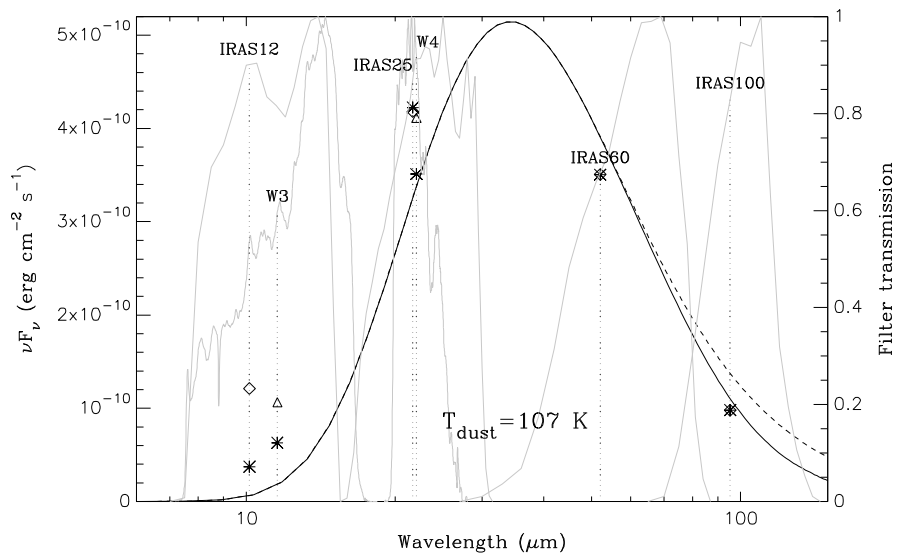

Fig. 14. Spectral energy distribution of the infrared emission in the central part of Ou4. Diamonds and triangles are IRIS and WISE data points, obtained from aperture photometry (Fig. 13). Grey lines show the transmission profiles of the IRAS and WISE broad-band filters, with vertical dotted lines indicating the effective wavelength. The solid line is the dust emission (single-temperature modified black-body model) fitted to the IRIS data points (diamonds), and asterisks are the corresponding modelled fluxes in each filter. The dashed line is the corresponding black-body model.

from dust grains. Assuming that all grains have the same size distribution and composition, the dust emission is a singletemperature modified black-body emission, that is a black-body emission multiplied by the dust emissivity (e.g., Draine 2004): $F_{v}=\Omega \times B_{v}\left(T_{\text {dust }}\right) \times\left[1-\exp \left\{-\tau_{\text {dust }}(v)\right\}\right]$, where $\Omega$ is a solid angle, $B_{v}\left(T_{\text {dust }}\right)$ is the black-body of temperature $T_{\text {dust }}$, and $\tau_{\text {dust }}(v)$ is the optical depth. The dust optical depth along the line of sight is defined by $\tau_{\text {dust }}(v)=\kappa_{\text {dust }}(v) \int \rho \mathrm{d} s$, where $\kappa_{\text {dust }}(v)$ is the dust opacity, and $\rho$ is the dust mass-density. We used the dust opacity corresponding to the Milky Way dust with $R_{V}=3.1$, computed for the carbonaceous/silicate dust model (Weingartner \& Draine 2001; Draine 2003). The low foreground-extinction can be neglected. We convolved $F_{v}$ with the transmission profiles of the broad-band filters ${ }^{10}$. We only fitted the IRIS data points to mitigate the variation of angular resolution with wavelength. Our best $\mathrm{fit}^{11}$ is obtained for a dust temperature of $107 \mathrm{~K}$. The decrease of dust emissivity with wavelength only affects the fluxes in the IRAS60 and IRAS100 bands (see dashed line in Fig. 14). The excess of emission in the IRAS 12 and $W 3$ bands may be due to additional emission from the background cloud. The excess of emission observed in the $W 4$ band compared with the model prediction is probably caused by the better angular resolution of WISE compared to IRIS. Our model predicts a maximum of dust emission around $34 \mu \mathrm{m}$.

In conclusion, the overall SED is typical of continuum emission from hot dust grains.

\section{Discussion and conclusions}

Summarising, one of the main results of this study is that the tip of the south lobe of Ou4 is shock ionised. This is clearly indicated by the spatial distribution of the atomic emission, with the higher ionisation species outwards and the lower excitation,

${ }^{10}$ We used the Filter Profile Service of the Virtual Observatory available at http://svo2 . cab.inta-csic.es/svo/theory/fps3/

11 The two other physical parameters are the diameter of the black-body region $(\equiv 2 d \sqrt{\Omega / \pi})$ of 89 AU for a distance, $d$, of $712 \mathrm{pc}$; and the dust surface mass $\left(\equiv \int \rho \mathrm{d} s\right)$ of $0.054 \mathrm{~g} \mathrm{~cm}^{-2}$. 
cooling post-shock gas inward, as well as by the observed line ratios and gas physical conditions.

The fact that this region is mainly ionised by collisions and not by photons does not require that the central source at the origin of the outflow has the high temperature that would be needed to excite ions such as $\mathrm{O}^{2+}$. Removing this constraint on the temperature of the central source may weaken the hypothesis that Ou4 is a PN. However, we do not have information about the ionisation mechanism of the inner regions of Ou4. It may well be that, in addition to shocks in the outermost region outflow, a small photoioinised core is present, as is the case of the PN KjPn 8 (López et al. 1995).

We estimated the probability of an apparent association between $\mathrm{H}$ II regions and PNe by cross-correlating the WISE catalogue of H II region (Anderson et al. 2014), which provides H II angular radii from $6^{\prime \prime}$ to 1.6 , with the catalogue of Galactic PNe of Kohoutek (2001). We identified 29 on 1510 PNe (i.e., 1.6\%) that are located inside HII regions in projection. The mean and standard deviation of their Galactic latitudes is -0.2 and 1.5 , with a maximum angular distance from the Galactic plane of 3.9. Therefore, the serendipitous association of $\mathrm{H}$ II regions and PNe only occurs close to the Galactic plane because of the high spatial density of the objects in this region of the sky. Since the Galactic latitude of Sh 2-129 is high $\left(b \approx 8^{\circ}\right)$, a fortuitous alignment with a PN appears as unlikely.

Given the apparent location of Ou4 in the sky, aligned with the young stellar cluster at the centre of the H II region Sh 2-129 and the striking correspondence between the $22 \mu \mathrm{m}$ and [O III] emission, it is reasonable to assume that $\mathrm{Ou} 4$ is an outflow launched some $90000 \mathrm{yr}$ ago from the massive triple system HR 8119. The location of the outflow, its radial velocities, and the extinction values are all consistent with this hypothesis. Additional support might come from the fact that the south bow-shock has an enhanced brightness in correspondence with a bright portion of Sh 2-129, as expected if it were impinging on a denser zone at the border of the H II region.

For comparison purposes, we estimated the kinetic energy of the Ou4 outflow. With a lobe size of $2.5 \mathrm{pc} \times 14.4 \mathrm{pc}$ in extent, the displaced mass is $M_{\text {disp }} \approx 2.1 M_{\odot}$ for a mean H II density of $1.7 \mathrm{~cm}^{-3}$ and $\mu=0.7$. With a shock propagating at $\approx 100 \mathrm{~km} \mathrm{~s}^{-1}$ the kinetic energy of the outflow is $K$ (outflow) $\approx 4 \times 10^{47} \mathrm{ergs}$, which is much lower than that of SNe. If the bipolar cavity were created by an episodic, collimated, bipolar jet/wind propagating at $2500 \mathrm{~km} \mathrm{~s}^{-1}$ (e.g., Steffen \& López 1998), the conservation of the kinetic-energy would imply a mass ejected by the driving source of about $M_{\text {ejec }} \approx 0.003 M_{\odot}$. With a dynamical time of $88000 \mathrm{yr}$, the required mass-loss rate would therefore be $3.8 \times 10^{-8} M_{\odot} \mathrm{yr}^{-1}$. This value can be compared with the mass-loss rate from the radiatively driven winds of the central massive stars. From the physical parameters of the stellar components $\mathrm{Aa}, \mathrm{Ab}$, and $\mathrm{B}$ of HR 8119 in Sect. 2.2 we estimate mass-loss rates of $\approx 2.5 \times 10^{-8}, \approx 0.6 \times 10^{-8}$, and $\approx 1.6 \times 10^{-8} M_{\odot} \mathrm{yr}^{-1}$ with terminal velocities of 2515,2508 , and $2517 \mathrm{~km} \mathrm{~s}^{-1}$ using the theoretical recipe of Vink et al. (2000). Therefore, the two-most massive stars of HR 8119 with a combined mass-loss rate of $4.1 \times 10^{-8} M_{\odot} \mathrm{yr}^{-1}$ and high terminal velocity can easily provide the required kinetic energy to drive Ou4. The larger separation of component Aa and B may explain the episodic phenomenon, whereas the close Ab companion could play a role in the outflow collimation.

In the alternative scenario that $\mathrm{Ou} 4$ is a PN, its overall morphology would very likely be the result of the expansion of a fast collimated wind from an as yet unidentified central source through a relatively dense circumstellar medium. If the cooling time of the shocked material (fast wind and/or ambient medium) is long enough, the fast collimated wind can inflate the observed thin lobes. The morphology of these lobes depends on several factors, mainly the opening angle of the fast outflow (Soker 2004). In addition, as discussed by Sahai et al. (1999) for the pre-PN Hen 401, the ambient density should decrease significantly with distance from the source to produce the cylindrical nebular morphology of the southern lobe. The presence of bowshocks and the bowl shape of the base of the southern lobe star are also consistent with models considering the inflation of bipolar lobes by fast collimated outflows seen nearly edge-on (Soker 2002; Balick et al., in prep.).

Studies of these types of collimated outflows in PNe and related objects often suggest an eruptive nature of the phenomenon, and that the central source is a binary system. It is appealing to assume that the outflow of Ou4 is produced in an outburst powered by mass accretion in a binary system, leading to a phenomenon such as an intermediate-luminosity optical transient (ILOT) as proposed for example for KjPn 8 (Boumis \& Meaburn 2013), other bipolar PNe (Soker \& Kashi 2012), or for massive $\left(M_{\text {ZAMS }} \sim 6-10 M_{\odot}\right)$ carbon-rich AGB, super-AGB, or post-AGB stars (Prieto et al. 2009).

Concluding, it is clear that the stellar source at the origin of the Ou4 outflow needs to be better constrained. A crucial information in this respect is its distance. In the future, we aim to precisely measure the proper motions of the outflow which, combined with the line-of-sight velocities and kinematical modelling, will provide a sound distance determination. A more sensitive imaging of the vicinity of the bright star HR 8119 with narrower filters centred on emission lines would be also valuable for detecting the origin of the outflow. In addition, we plan to extend this study to other wavelength domains to better constrain the nature of this unique giant outflow.

Acknowledgements. R.L.M.C. and N.G. equally contributed to this work. We thank the referee John Bally for many useful suggestions that helped to improve our manuscript. We are grateful to the time allocation committee (CAT) for awarding us IAC Director Discretionary Time at the WHT and INT. The higher resolution spectra were secured during ING service time. We thank the ING staff, and in particular Javier Mendez, Raine Karjalainen, and the ING students for imaging attempted on an additional night, which could not be used because of the strong moonlight, which prevented detection of the faint nebula. R.L.M.C. acknowledges funding from the Spanish AYA2007-66804 and AYA2012-35330 grants. We thank Stéphane Zoll for his [O III] image of Ou4. Finally, we are very grateful to Gabriel Perez at the IAC for the careful edition of the colour image in Fig. 4. This research has made use of Aladin, and of the SIMBAD database, operated at CDS, Strasbourg, France. This publication makes use of data products from the Wide-field Infrared Survey Explorer, which is a joint project of the University of California, Los Angeles, and the Jet Propulsion Laboratory/California Institute of Technology, funded by the National Aeronautics and Space Administration.

\section{References}

Acker, A., Boffin, H. M. J., Outters, N., et al. 2012, Rev. Mex. Astron. Astrofis., 48, 223

Anderson, L. D., Bania, T. M., Balser, D. S., et al. 2014, ApJS, 212, 1

Bertelli, G., Bressan, A., Chiosi, C., Fagotto, F., \& Nasi, E. 1994, A\&AS, 106, 275

Bertin, E., \& Arnouts, S. 1996, A\&AS, 117, 393

Bertin, E., Mellier, Y., Radovich, M., et al. 2002, in Astronomical Data Analysis Software and Systems XI, eds. D. A. Bohlender, D. Durand, \& T. H. Handley, ASP Conf. Ser., 281, 228

Bessell, M. S., \& Brett, J. M. 1988, PASP, 100, 1134

Blitz, L., Fich, M., \& Stark, A. A. 1982, ApJS, 49, 183

Bonnarel, F., Fernique, P., Bienaymé, O., et al. 2000, A\&AS, 143, 33

Boumis, P., \& Meaburn, J. 2013, MNRAS, 430, 3397

Brocklehurst, M. 1971, MNRAS, 153, 471

Cohen, J. G., Persson, S. E., Elias, J. H., \& Frogel, J. A. 1981, ApJ, 249, 481 
R. L. M. Corradi et al.: Gas physical conditions and kinematic of the giant outflow Ou4

Condon, J. J., Broderick, J. J., Seielstad, G. A., Douglas, K., \& Gregory, P. C. 1994, AJ, 107, 1829

Cutri, R. M., Wright, E. L., Conrow, T., et al. 2012, Explanatory Supplement to the WISE All-Sky Data Release Products, Tech. rep.

De Robertis, M. M., Dufour, R. J., \& Hunt, R. W. 1987, JRASC, 81, 195

Deharveng, L., Schuller, F., Anderson, L. D., et al. 2010, A\&A, 523, A6

Draine, B. T. 2003, ApJ, 598, 1026

Draine, B. T. 2004, in The Cold Universe, eds. A. W. Blain, F. Combes, B. T.

Draine, D. Pfenniger, \& Y. Revaz (Springer), 213

Draine, B. T., \& McKee, C. F. 1993, ARA\&A, 31, 373

Finkbeiner, D. P. 2003, ApJS, 146, 407

Fitzpatrick, E. L. 1999, PASP, 111, 63

Georgelin, Y. P., \& Georgelin, Y. M. 1970, A\&AS, 3, 1

Hartigan, P., Raymond, J., \& Hartmann, L. 1987, ApJ, 316, 323

Hartigan, P., Raymond, J., \& Meaburn, J. 1990, ApJ, 362, 624

Hiltner, W. A. 1956, ApJS, 2, 389

Høg, E., Fabricius, C., Makarov, V. V., et al. 2000, A\&A, 355, L27

Kohoutek, L. 2001, A\&A, 378, 843

López, J. A., Vázquez, R., \& Rodríguez, L. F. 1995, ApJ, 455, L63

Mamajek, E. E., Meyer, M. R., \& Liebert, J. 2002, AJ, 124, 1670

Mason, B. D., Wycoff, G. L., Hartkopf, W. I., Douglass, G. G., \& Worley, C. E. 2001, AJ, 122, 3466

Mason, B. D., Hartkopf, W. I., Gies, D. R., Henry, T. J., \& Helsel, J. W. 2009, AJ, 137, 3358

Meyer, M. R., Calvet, N., \& Hillenbrand, L. A. 1997, AJ, 114, 288

Miville-Deschênes, M.-A., \& Lagache, G. 2005, ApJS, 157, 302

Nazé, Y., Chu, Y.-H., Guerrero, M. A., et al. 2002, AJ, 124, 3325
Oke, J. B. 1990, AJ, 99, 1621

Patel, N. A., Goldsmith, P. F., Heyer, M. H., Snell, R. L., \& Pratap, P. 1998, ApJ, 507, 241

Pecaut, M. J., \& Mamajek, E. E. 2013, ApJS, 208, 9

Prieto, J. L., Sellgren, K., Thompson, T. A., \& Kochanek, C. S. 2009, ApJ, 705 1425

Reipurth, B., \& Bally, J. 2001, ARA\&A, 39, 403

Reynolds, R. J. 1977, ApJ, 216, 433

Sahai, R., Bujarrabal, V., \& Zijlstra, A. 1999, ApJ, 518, L115

Santander-García, M., Corradi, R. L. M., Balick, B., \& Mampaso, A. 2004, A\&A, 426, 185

Shaw, R. A., \& Dufour, R. J. 1995, PASP, 107, 896

Skrutskie, M. F., Cutri, R. M., Stiening, R., et al. 2006, AJ, 131, 1163

Soker, N. 2002, ApJ, 568, 726

Soker, N. 2004, A\&A, 414, 943

Soker, N., \& Kashi, A. 2012, ApJ, 746, 100

Steffen, W., \& López, J. A. 1998, ApJ, 508, 696

Tenorio-Tagle, G. 1979, A\&A, 71, 59

Viironen, K., Greimel, R., Corradi, R. L. M., et al. 2009, A\&A, 504, 291

Vink, J. S., de Koter, A., \& Lamers, H. J. G. L. M. 2000, A\&A, 362, 295

Weingartner, J. C., \& Draine, B. T. 2001, ApJ, 548, 296

Wilson, R. E. 1953, General catalogue of stellar radial velocities, 601 (Washington: Carnegie Institution of Washington)

Wright, E. L., Eisenhardt, P. R. M., Mainzer, A. K., et al. 2010, AJ, 140, 1868

Yorke, H. W., Tenorio-Tagle, G., \& Bodenheimer, P. 1983, A\&A, 127, 313

Zirm, A., \& Rica, F. 2012, Inf. Circ. IAU Double Stars, 176 